\title{
REAL-TIME NAVIGATION SYSTEM IMPLEMENTATION WITHIN THE SCOPE OF 3D GEOGRAPHIC INFORMATION SYSTEMS FOR MULTI STOREY CAR PARK
}

\author{
B. Yenigun ${ }^{\mathrm{a}}$, I. R. Karas ${ }^{\mathrm{b}}$, E. Demiral ${ }^{\mathrm{c}}$ \\ aKarabuk University, Engineering Faculty, Karabuk, Turkey - bernagunes@karabuk.edu.tr \\ ${ }^{b}$ Karabuk University, Engineering Faculty, Karabuk, Turkey - ismail.karas@karabuk.edu.tr \\ cKarabuk University, Engineering Faculty, Karabuk, Turkey - emrullahdemiral@karabuk.edu.tr
}

KEY WORDS: Wireless Sensor Network, Indoor Parking, Navigation, RFID, Ultrasonic Sensor

\begin{abstract}
:
Today, a large increase in the number of vehicles in traffic with population growth and economic development are observed. This increase brings with it many problems. One of these problems is the parking problem as a result of the need to park the vehicle. Impractical and inadequate parking areas have a negative impact on drivers. To minimize these drawbacks, car parking is intended to develop a system to come to the aid of users. This system is planned to be developed which can be applied to indoor and multi-storey parking structure. Considered in this study, in the garage, drivers are intended to be directed as soon as possible in place of the convenient parking. However, the amount of fuel spent looking for parking tool that are caused by air and noise pollution, and to search for a parking lot when looking for a place to park the lost time, the resulting stress is minimized, it is expected that.
\end{abstract}

\section{INTRODUCTION}

The number of vehicles in traffic along with economic development and population growth are increasing day by day. According to data of 2016 TUIK (Turkey Statistical Institute), registered to traffic by the end of January, there are a total of 20 million 98 thousand 994 motor vehicles and cars constitute $53 \%$ of these vehicles. With the presence of so many vehicles are greater problems arise if thought to be more than the duration of the parking of vehicles. These problems are the lack of parking spaces for drivers; it is also due to incorrect parking. Therefore, the studies for parking have gained momentum and every day the best parking strategy is searched for. Now having built large structures in the underground garage, contribute a little bit to get a solution to this traffic. The purpose of the planned system is to reduce the amount of fuel and time that they spend to search for empty parking lots. Less fuel consumption will minimize air and noise pollution caused by vehicles. Moreover, this system will eliminate long vehicle queues that occurring because of the traffic they create while searching empty parking lot. The stress of the driver to find an empty parking lot is expected to decrease with and the parking rates are expected to increase this system. In this case the quality of life will improve. What day and time it is more intensity in the car park, it will be determined with this system and best parking strategy can be developed for parking. If one of the biggest problems we face today also considered to be security, with this planned system, the credentials of the vehicles entering the parking lot will be recorded and can be identified that could threaten the security tools.

The operation of this system is planned to be as follows. - Parking on the ground, on which floors, there is an information board showing how empty parking lot. Driver goes to the floor will parking based on this information.
- Each floor entrance, there are indicators that show information about the current free parking areas in the floor. Driver goes to the floor with the empty parking lot according to the information in the information display.

- Allocated parking for each vehicle in the parking lot, occupancy and vacancy status, by means of the sensor is monitored.

- When the driver came to the floor where the empty parking space, the vehicle's location is determined and the closest to the vehicle empty parking lot, it is determined by the system to be installed.

-The driver, reaches into empty parking lot follow the signs of led demonstrators which located at the beginning of each corridor.

Parking guidance system is an open technology developed and implemented in different ways in many countries. This system is applied in more open areas. This is because of the difficulties that may arise in closed areas to determine the vehicle's position. Studies to determine the location in a confined space is growing every day. With these developments, the position detection experiment in an indoor parking garage has accelerated. Routing the vehicle is detected in the parking position is performed in different ways. For example, in 2011 Silva, has designed a system that provides information about lot in the parking area by helping visually to the driver (Figure 1). The driver prefers a corridor, based on knowledge of monitor empty parking in the car park entrance. When the driver enters the parking area, updated information on the monitor. Wireless sensor network in the parking lot comprises magnetic sensors which control the fullness information of the parking lots and it is located on all parking areas. This Information is 
transmitted to the server and processed information is sent back to the screen in the hall (Silva , 2011).

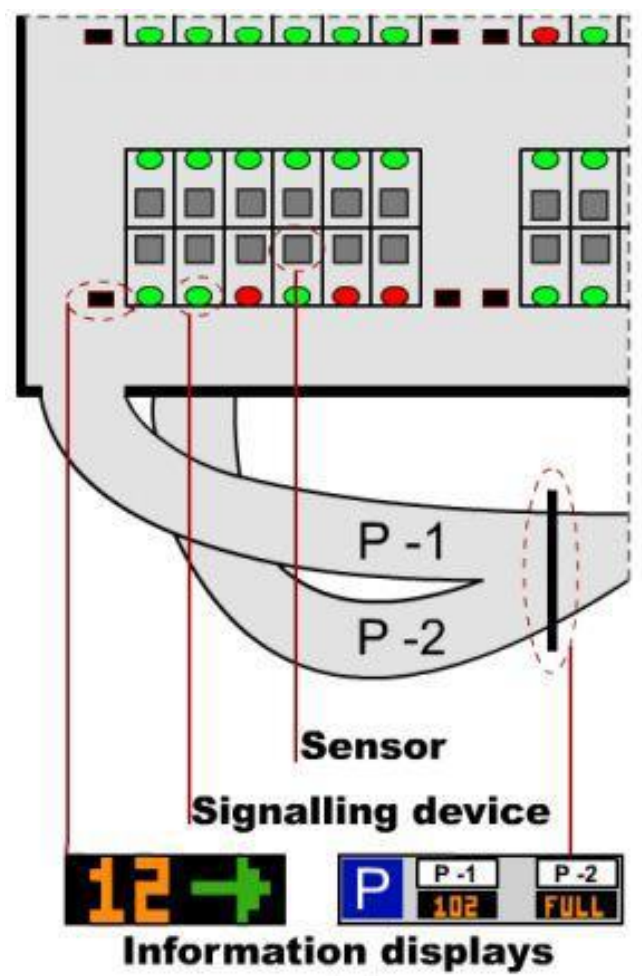

Figure 1. System components

Kianpisheh in 2012 will help drivers find empty parking lot in a short time, a new parking system called SPS is designed (Figure 2). This system is able to detect both inappropriate parking and parking occupancy information. The system is applied to 5 corridors on each floor and a 4-storey car park with 100 parking. The driver can see how many empty parking lots which are on the floor via LED indicators on the input. When the driver arrived about the floor, it looks at interior signs hanging from the ceiling of each hallway. Each internal mark shows both the number of empty parking lot and the direction of the corridor with empty lot. There are red, yellow, blue and green LED lights each in the parking lot. Green means the space is vacant, red means the space is occupied, blue means the space is assigned for handicapped drivers and yellow means it has been reserved space. In this study, ultrasonic sensors, LED indicators, internal and external display panels, field control unit (Zone Control Unit), the central control unit (Central Control Unit), network switches, telephone cable and management software is used. Ultrasonic sensors, transmit the status message to $\mathrm{ZCU}$ through the phone cord, ZCU transmits information to the CCU via CAT5 cable. CCU processes information and sends commands to the ZCU and LED indicators. ZCU connects to the internal display and ultrasonic sensor using the RS-485 port and it communicates with the CCU via network switches and LAN connections (Kianpisheh, 2012).

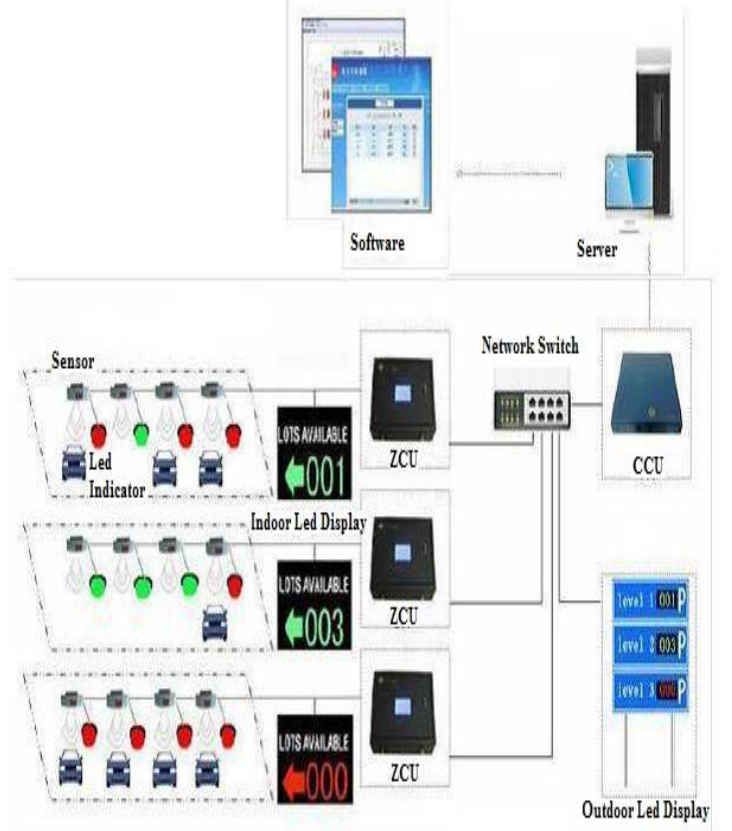

Figure 2. Prototype of intelligent parking system

Sayeeraman, in 2012, using wireless technology, focused on the reservation and vehicle parking. The system contains three nodes including infrared sensors and ZigBee interface with an empty parking lot monitoring module has PIC microcontroller, parking lot reservation module including GSM modem and security module including a set of rules that allow security (Figure 3). In this study, drivers can reserve a parking space by SMS. When the coordinator system receives the SMS from the user, it will start to check whether there is any vacancy in the parking lot. If there is empty parking lot, it sends a message containing the login password to the driver. When the driver reaches the parking space, barrier opens if the driver enters the correct password. As used herein, infrared sensors can detect $24 \times 7$. If the sensor detects a vehicle in the parking lot, it tells the microcontroller the presence of a vehicle. The microcontroller sends in order status information to the ZigBee nodes. The ZigBee node transmits the status information to the end ZigBee node which is interfaced with a coordinator system at the entrance of the parking and the parking lot status is updated in the database system. UART communication between ZigBee node and the microcontroller is carried out. Access control system has a GSM modem and ZigBee node. Dot net framework is used to create access system. Also, the system has a user interface formed by ASP.NET. The driver is able to reserve parking with this interface (Sayeeraman, 2012). 


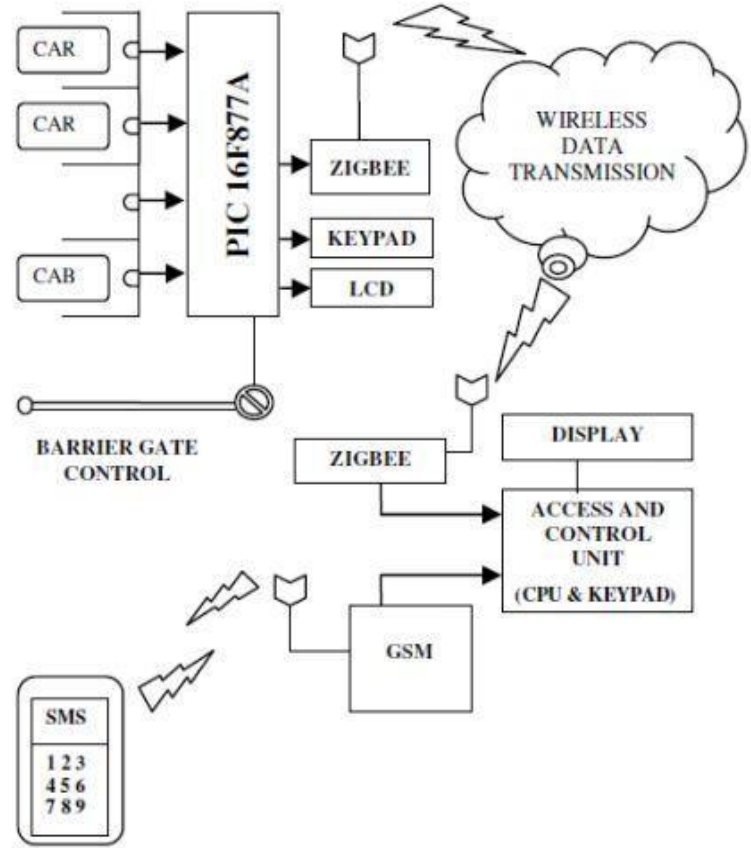

Figure 3. Block diagram of operation

In 2013, Gódor has designed a system intended that the driver who goes into indoor parking to be directed to the appropriate parking lot with smart phone. The driver selects one of the preferences such as the nearest parking lot, family parking or the closest parking area favorite shop via smart phone at the car park entrance. By the this choice is determined the most convenient parking lot is determined and parking lot coordinates and maps is transmitted to the driver's Smartphone. By following this map, which is updated according to the position of the driver, the driver reaches the appropriate parking lot (Figure 4). Here, parking occupancy information is collected by the ultrasonic sensors. Central Server System consists of Communication Infrastructure, Wi-Fi Access Points (AP), and Client Devices. To estimate the Driver's position, the signal-based fingerprint algorithm was used. Central Service shows the fingerprints stored in the database and the map of the parking lot. In Database, both fingerprints of drivers and fingerprints of pedestrians are stored. A simple algorithm has been used to decide which one of these should use. The users entering the garage at the car entrance will use the in vehicle database, while the users coming from inside the building will utilize the pedestrian fingerprint database (the fingerprints were recorded with a device carried by a pedestrian). The server also shows the parking occupancy information by means of ultrasonic sensors. Wi-Fi infrastructure used to detect position consists of 10 Access Point 3 switches and a central server. Server, collects Wi-Fi signal strength information of the visiting mobile devices via access points, calculates the positions of the mobile nodes to be tracked and sends back the calculated position information to the visiting device via the Wi-Fi infrastructure. Wi-Fi Access Point collects the necessary data (RSS information of each client device) for the position estimation algorithm, forwards the collected data to the central server and connects the client devices to the background infrastructure. It has also been used as a method for determining position: By measuring and collecting RSS data of all the APs, fingerprint database of the given area is built. APs are set with $4 \mathrm{~m}$ grid distance. Visiting Device measures every signal coming from AP continuously and it sends to the server this measurements. Visiting Device measures every signal coming from AP continuously and it sends to the server this measurement (Gódor, 2013).

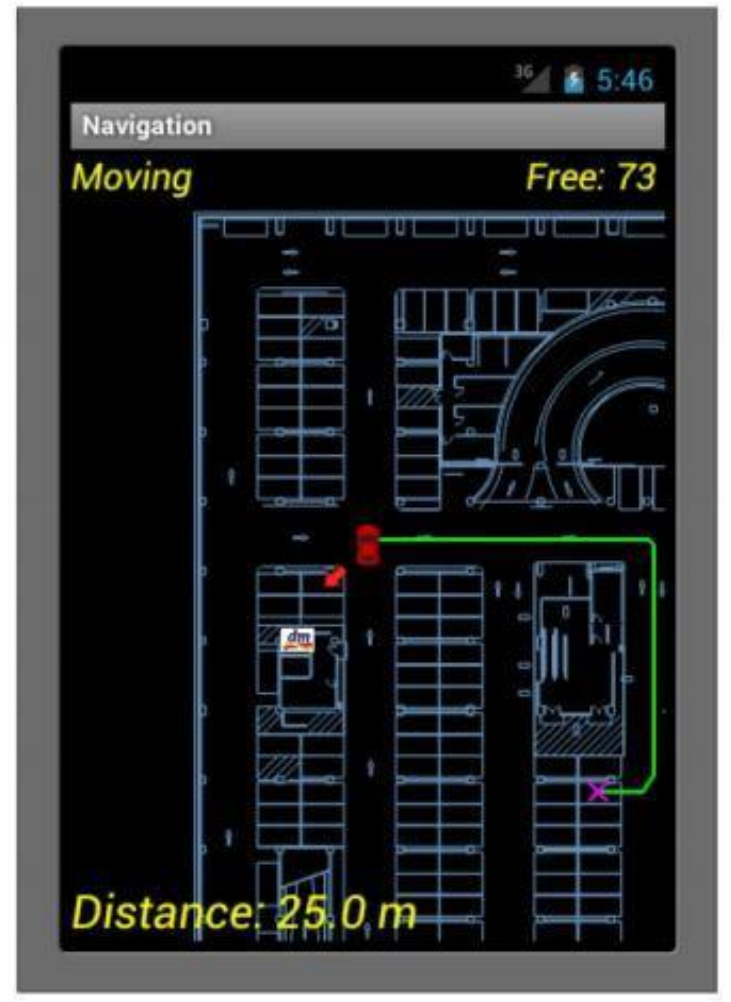

Figure 4. Navigation interface

\section{MATERIALS AND METHODS}

The planned system, to make real-time network analysis, a real-time remote control system is required. This control is intended to cover in the sensors and the server. At the top of each parking lot will have a sensor and the sensors will transmit to the server parking occupancy information. The server will check LED indicators that will route the user with the information received; also it will transfer this information to the database. It is necessary to determine the first empty parking place for the establishment of such a system. For this, it is considered to design a sensor circuit. The most important element of this circuit will be Arduino board, ultrasonic sensor and RGB (red, green, blue) LEDs. 


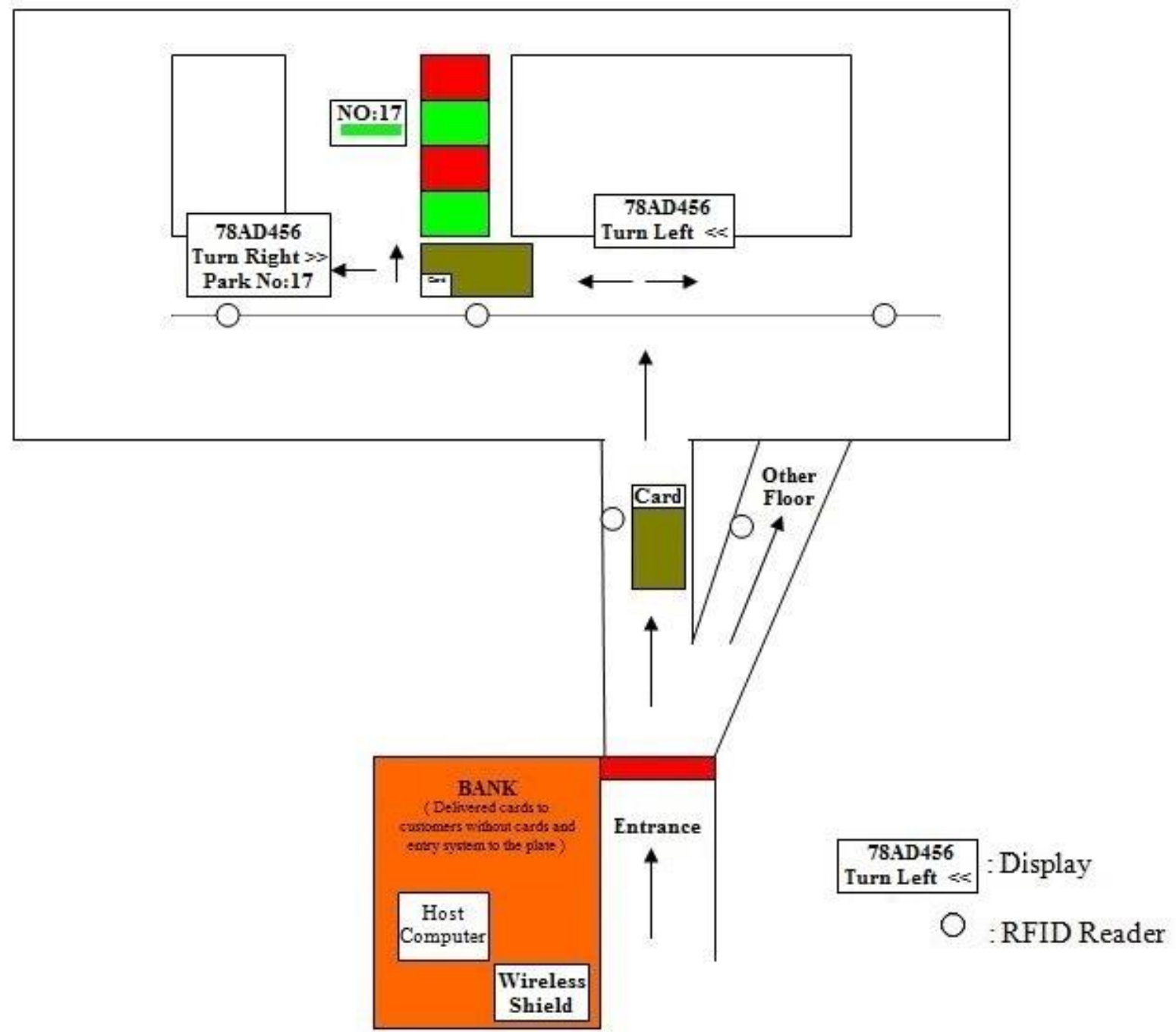

Figure 5. Operation of the system

To determine the parking occupancy information, an ultrasonic sensor is considered. Ultrasonic sensors are sensors that emit sound waves and they can determine the distance by calculating the time it takes to get back and hit obstacles (vehicle or place) (Carullo, 2001).

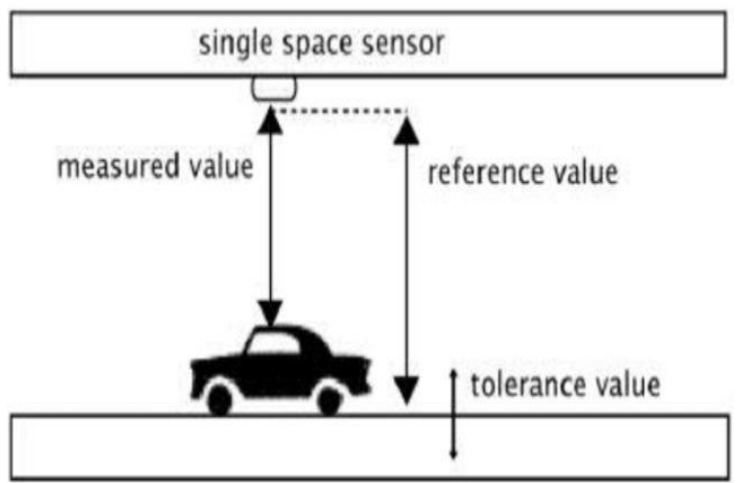

Figure 6. The operation of the ultrasonic sensor (Lynn, 2009)
Information whether the vehicle in this area will be transmitted to the server via wireless shield. This communication is planned to be carried out in a wireless form. In this communication, low-speed Personal Area Network standard ZigBee wireless communication technology is intended to be used. ZigBee is Wireless Sensor Network (WSN) technology based on IEEE 802.15.4 standards (Kinney, 2003). The incoming data to the server with wireless will be stored by processing. It is planned interface for parking management on the computer. In this interface, the parking model and the parking lot information will be shown.

\subsection{Determining the Location of the Vehicle}

The RFID (Radio Frequency Identification) technology is thought to be appropriate to determine the location of the vehicle in the parking because it has lot of advantages. RFID is a term used to define wireless non-contact use of radio frequency electromagnetic fields to transfer identification data of an object for the purposes of identifying and tracking (Khong and White, 2005). There are two basic components of RFID system, including readers and carriers. There are reader fixed, carrier active or carrier fixed, reader active form (Demiral, 2014). In 
operation, it is planned to set RFID readers at regular intervals on the ceiling of the parking garage to determine the location of vehicle also RFID tags on vehicles of users. When the vehicle with the tag enters into the domain of RFID reader, the electromagnetic wave exchange begins between the reader and tag. After this exchange, the reader transmits the server received the information.

\subsection{Production of the Car Park of 3D Network Model, Acquisition Attributes of Information, in Relation with Each Other Be Combined Spatial Database}

Developing targeted systems will use three-dimensional (3D) data models of the parking garage in network analysis and navigation applications. This model is 3D Road Network Model. Therefore, first of all, to the car park which is subject to project, it will need to produce this model. In the production of a 3D topological Road Network Model, "Multidirectional Scanning for Line Extraction" (MUSCLE Model) method by developed Karas (2007) will be used. This method is a digital image processing algorithm, in the general sense, was developed in order to vectorize raster images with straight lines (Karas, 2007).

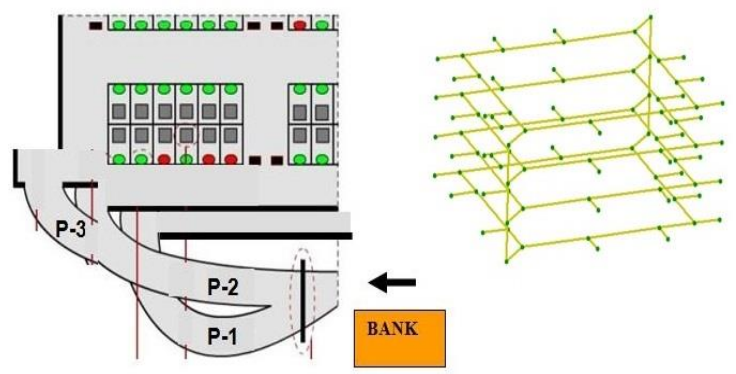

Figure 7. Example floor plan and network structure

\subsection{Modification of the Navigation Software to Help with Making Network Analysis and Development of Additional Modules for Vehicles}

Done to determine the position of the vehicle, then these data are transmitted to the server, it is necessary to identify the location of empty parking the closest to the vehicle's location. After the data is processed on the computer, the road map of empty parking lot close to the vehicle's position will be created using the shortest path algorithm. It is planned to develop software for. The algorithm works is considered as follows: As soon as the driver's position is detected, the algorithm starts operating. The algorithm will guide drivers to empty parking lot toward the entrance starting from at the end of the lot of the park. If the user went to the wrong direction, the algorithm creating a new roadmap will find the nearest free parking to the vehicle's position.

\subsection{Development of Guidelines Module Software}

Developed software will help set the users nearest empty parking space. This software aims at guiding the driver to empty parking space nearest to the vehicle with created the road map. For this orientation, the LEDs to be placed in the crossroads of the parking lot indicators will be used. The driver will reach the parking lot by following direction information generated by the software from the server via LED indicators.

\subsection{The Establishment of System Architecture and Communication Infrastructure and the Development of Communication-Related Software}

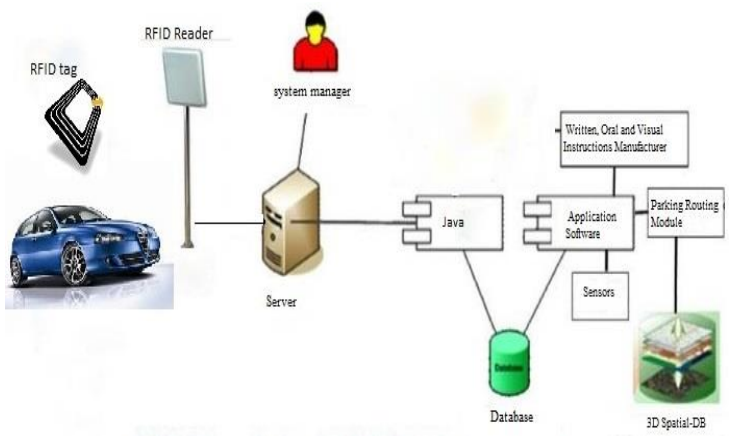

Figure 8. In the project phase will emerge estimated components of the system architecture

Architecture of the system will be created at the project stage is estimated to be as described above.

\subsection{Testing the System with Different Scenarios, Improving by Test Results}

Vehicle Routing System will be tested under different scenarios. Situations will be taken into consideration such as the driver may enter the false road outside the routing of system, may miss by not see the proposed park area or may park the vehicle to another parking area instead of the parking area identified by the system. Different combinations that may occur will emerge more clearly in the future stages of the study. Consequently, the necessary improvements to be made on the system and possible errors will be eliminated. Then, using the developed Positioning and Navigation System, in real time will be implemented in the field.

\section{RESULTS}

This system which will direct drivers to empty parking spaces will provide great convenience to vehicle users. Drivers searching for parking will consume less fuel thanks to this system will also have to save time. This system differs from already existing parking management system, the driver continued guidance until you reaches the empty parking lot and on the move the driver's position information is constantly updated. Also vehicle movements in the car park can be saved on the server. These data will pave the way for new locational analyzes. These data by analyzing can be used in solving many different problems such as Improving the parking lot, detection and elimination of them that if there are situations or sings causing to the mistakes for the drivers. For the future, the study will provide obtaining positive outcomes such as improvement of parking, tracking and organization of the drivers in the parking lot. Later in the study, the project is intended to develop the mobile device so that it can also be used. In addition, drivers, via SMS or the Internet, can reserve free parking spaces are planned to be desired date and time. With advances in technology, this project is proposed to be integrated into a 
wide array system. Appropriate uses of the system may be investigated in terms of parking security.

It should be considered as an advantage for the proposed system in this study that it has lower cost compared to smart parking system using lift (Urganc1, 2016) and also it can be usable for the majority of existing storey car park.

\section{ACKNOWLEDGEMENT}

This study was supported by Karabük University, Department of Scientific Research Projects (Project No: KBÜ-BAP-16/1-YL-141) research grant. We are indebted for its financial support.

\section{REFERENCES}

Carullo, A., \& Parvis, M., 2001. An ultrasonic sensor for distance measurement in automotive applications. IEEE Sensors journal, 1(2), 143-147.

Demiral, E., 2014. RFID-based indoor positioning system design within the scope of three dimensional (3d) geographic information systems. Master's Thesis, Karabuk University, Karabuk.

Gódor, G., Huszák, Á., \& Farkas, K., 2013. Intelligent indoor parking. In: Proceedings of the 1st Global Virtual Conference, Stip, Macedonia.

Karaş, İ. R., 2007. Evaluating Topological Relationships of the Objects in 3D GIS and Network Analysis. $\mathrm{PhD}$ Thesis, Yildiz Technical University, Istanbul, 12-15.

Khong G, White S, 2005. Moving right along: Using RFID for Collection Management at the Parliamentary Library. Information-Online 12 th Exhibition \& Conference, Sydney.

Kianpisheh, A., Mustaffa, N., Limtrairut, P., \& Keikhosrokiani, P., 2012. Smart parking system (SPS) architecture using ultrasonic detector. International Journal of Software Engineering and Its Applications, $6(3), 55-58$.

Kinney, P., 2003. Zigbee technology: Wireless control that simply works. In: Communications design cenderence (Vol. 2, pp. 1-7).

Lynn, C., 2009. Parking Guidance Systems. In: Parking Consultants International, Sydney.

Sayeeraman, A., \& Ramesh, P. S., 2012. ZigBee and GSM based secure vehicle parking management and reservation system. Journal of Theoretical and Applied Information Technology, 37(2), 199-203.

Silva, D., Bartolomeu, P., \& Fonseca, J., 2011. Wireless parking lot monitoring and guidance. In Emerging Technologies \& Factory Automation (ETFA), IEEE 16th Conference on (pp. 1-4). IEEE.
Urganc1, S., 2016. Youtube "Akıllı Otopark", https://www.youtube.com/watch?v=BSsTyX5v6Ds (02 Aug. 2016) 